\title{
Studies on Some Salicylhydroxamate Complexes of Aryltellurium(IV)
}

\author{
SONU CHAUHAN, SAPANA GARG and K.K.VERMA* \\ Department of Chemistry, Maharshi Dayanand University, Rohtak-124001, India \\ vermakk123@rediffmail.com
}

Received 19 December 2015 / Accepted 11 January 2016

\begin{abstract}
Twelve new salicylhydroxamate complexes of the type $\mathrm{ArTeCl}_{2} \cdot \mathrm{SHA}, \mathrm{ArTeCl}$.(SHA) ${ }_{2}$, $\mathrm{Ar}_{2} \mathrm{TeCl}$.SHA and $\mathrm{Ar}_{2} \mathrm{Te}$.(SHA) ${ }_{2}$ (where $\mathrm{Ar}=p$-methoxyphenyl, $p$-hydroxyphenyl, 3-methyl-4hydroxyphenyl; SHA = salicylhydroxamate) have been synthesized by reactions of aryltellurium(IV) trichlorides and diaryltellurium(IV) dichlorides with potassium salicylhydroxamate. The aryltellurium(IV) hydroxamates have been characterized on the basis of elemental analyses, conductance measurements in DMSO, infrared and proton magnetic resonance studies. The spectral studies conjointly predicts the bidentate $\left(O, O^{\prime}\right)$ nature of hydroxamates to yield the penta and hexacoordinated tellurium complexes. The biological activities of these complexes were evaluated against some pathogenic bacteria and fungi organisms.
\end{abstract}

Keywords: Salicylhydroxamate, Aryltellurium(IV), Diaryltellurium(IV), Antibacterial, Antifungal activites

\section{Introduction}

The hydroxamic acids, with general formula $\mathrm{RCON}(\mathrm{H}) \mathrm{OH}$, are well known class of compounds due to their strong chelating properties. Hence they are involved in many biochemical processes such as iron transport phenomena ${ }^{1}$, inhibition of enzymatic activity of urease $^{2,3}$, Alzheimer's Amyloid precursor protein $\alpha$-secretase ${ }^{4}$ and some matrix metalloproteinases ${ }^{5}$. They also display antibacterial, anti inflammatory and anti-asthmatic behaviour $^{6,7}$ and have been utilized in the design of therapeutic targets for cancer ${ }^{8,9}$, Alzheimer's disease ${ }^{10}$, haemochromatosis ${ }^{11,12}$ and malaria ${ }^{13}$. Hydroxamic acids have been extensively studied as bioligands forming chelate complexes with numerous metals ${ }^{14-17}$ including organotin complexes ${ }^{18,19}$.

Salicylhydroxamte $\left(\mathrm{SHA}^{-}\right)$can chelate via a number of possible coordination modes, not only the $O, O$ - mode, but also $N O$-with the phenolato oxygen as $O$-donor, while most instance the hydroxamates are bound via $O, O$ - chelating ${ }^{20-27}$.

Also, aryltellurium(IV) trichlorides are known ${ }^{28-41}$ to behave as lewis acids and form complexes with several N- , O- and S- donor bases. The, diaryltellurium(IV) dichlorides are 
also reported to act as acceptors but much weaker than aryltellurium(IV) trichlorides ${ }^{42-44}$. In view of this, it was thought desirable to study the reactions of potassium salicylhydroxamate with some aryltellurium trichlorides and diaryltellurium dichlorides. In this paper, we report the synthesis, characterization and antimicrobial studies on some new hydroxamates of the type $\mathrm{ArTeCl}_{2}$.SHA, ArTeCl.(SHA) $)_{2}, \mathrm{Ar}_{2} \mathrm{TeCl}$.SHA and $\mathrm{Ar}_{2} \mathrm{Te}$.(SHA) $)_{2}$ where, $\mathrm{Ar}=p$-methoxyphenyl, $p$-hydroxyphenyl and 3-methyl-4-hydroxyphenyl and SHA = salicylhydroxamate.

\section{Experimental}

All preparations were carried out under an atmosphere of dry nitrogen and the solvents used were purified by standard method ${ }^{45,46}$ before use. The purity of compounds was checked by TLC using Silica gel-G (Merck). Melting points were determined in open capillary tube and are uncorrected.

Carbon, hydrogen and nitrogen analyses were obtained microanalytically from SAIF, Panjab University Chandigarh on a ThermoFinnigan CHNS analyser. Conductivity was measured in DMSO at $25 \pm 2{ }^{\circ} \mathrm{C}$ with a microprocessor based conductivity bridge type MICROSIL.

IR spectra were recorded in $\mathrm{KBr}$ pellets at SAIF, Panjab University Chandigarh on a F.T. Infra-Red spectrophotometer Model RZX (Perkin Elmer). ${ }^{1} \mathrm{H}$ NMR spectra were recorded in DMSO-d $\mathrm{d}_{6}$ using TMS as an internal reference on BRUKER AVANCE II 400 NMR spectrometer. The antimicrobial screening was carried out by tube dilution method at Department of Pharmaceutical Sciences, M. D. University, Rohtak.

\section{Preparation of aryltellurium(IV) trichlorides and diaryltellurium(IV) dichlorides}

p-Methoxyphenyltellurium(IV)trichloride ${ }^{47,48}$, bis( $p$-methoxyphenyl)tellurium(IV) dichloride ${ }^{48,49}$, $p$-hydroxyphenyltellurium(IV) trichloride ${ }^{50}$, bis ( $p$-hydroxyphenyl) tellurium (IV) dichloride ${ }^{50}, 3$ methyl-4-hydroxyphenyltellurium(IV) trichloride $^{51}$ and bis(3-methyl-4-hydroxyphenyl)tellurium(IV) dichloride ${ }^{51}$ were prepared by the reactions of tellurium tetrachloride with corresponding arenes i.e. anisole, phenol, $o$-cresol respectively, by the methods reported in the literature.

\section{Preparation of potassium salicylhydroxamate (KSHA)}

Potassium salicylhydroxamate was prepared in two steps as follows:

\section{Preparation of ethyl ester of salicylic acid ${ }^{45}$}

To 0.25 mole of salicylic acid, excess (up to 2.5 moles) of ethyl alcohol and $1 \mathrm{~mL}$ of conc. $\mathrm{H}_{2} \mathrm{SO}_{4}$ in separate reaction flasks were added. The reactants were refluxed for about $3 \mathrm{~h}$ till whole of the acid dissolved in ethanol. The contents were cooled and transferred to $60 \mathrm{~mL}$ of water in a separating funnel. The lower layer of ester was removed and was washed with saturated sodium bicarbonate until the effervescence ceased. Finally washed with water and dried over anhydrous sodium sulphate.

\section{Preparation of potassium salt of hydroxamic acid}

The potassium salt was prepared by the method reported by Houser et al., ${ }^{52}$. Cooled solution of $\mathrm{KOH}$ (28.05 g in $70 \mathrm{~mL}$ methanol) was added to methanolic solution of hydroxylamine ( $23.27 \mathrm{~g}$ in $120 \mathrm{~mL}$ ) with constant shaking and cooling. The mixture was allowed to cool for 24 hours in an ice bath to ensure complete precipitation of $\mathrm{KCl}$, which was removed by filteration. To this filtrate was added $25 \mathrm{~mL}$ of ethyl ester of salicylhydroxamic acid. The reaction mixture was kept in air tight flask at room temperature for 2-3 days to yield the fine crystals of potassium salicylhydroxamate, which were filtered and dried in air. Yield $80 \%$, m. pt. $260-270{ }^{\circ} \mathrm{C}$. 


\section{Preparation of aryltellurium(IV) salicylhydroxamates}

Aryltellurium(IV) trichlorides, $\mathrm{ArTeCl}_{3}(\mathrm{Ar}=p$-methoxyphenyl, $p$-hydroxyphenyl, 3-methyl-4-hydroxyphenyl), when treated with potassium salicylhydroxamate in different molar ratios, yield $\mathrm{ArTeCl}_{2}$. $\mathrm{SHA}$ and $\mathrm{ArTeCl}$.(SHA) $)_{2}$ type complexes.

\section{$\mathrm{ArTeCl}_{2} \cdot \mathrm{SHA}$}

A warm saturated methanolic solution of potassium salicylhydroxamate $(0.38 \mathrm{~g}, 2 \mathrm{mmol})$ was added dropwise to a solution of aryltellurium(IV) trichloride $(2 \mathrm{mmol})$ in chloroform/methanol. An immediate precipitation of $\mathrm{KCl}$ resulted which was removed by filteration. The filterate was further refluxed for 3-4 hours to precipitate out any $\mathrm{KCl}$ and clear solution was then concentrated to about one third of original volume and kept overnight to yield crystalline product. This was filtered, washed with chloroform and dried in a vacuum esiccators over $\mathrm{P}_{4} \mathrm{O}_{10}$.

\section{$\mathrm{ArTeCl} .(\mathrm{SHA})_{2}$}

The saturated solution of aryltellurium(IV) trichloride $(2 \mathrm{mmol})$ in chloroform/methanol was added dropwise with constant stirring to a saturated methanolic solution of potassium salicylhydroxamate $(0.76 \mathrm{~g}, 4 \mathrm{mmol})$. An immediate change in colour with precipitation of $\mathrm{KCl}$ took place, which was removed by filteration. The contents were refluxed for 3-4 hours and then clear solution concentrated to about one third of original volume and left overnight to get coloured crystalline product, which was filtered, washed with chloroform and dried in a vacuum esiccators over $\mathrm{P}_{4} \mathrm{O}_{10}$.

\section{Preparation of diaryltellurium(IV) salicylhydroxamates}

Diaryltellurium(IV) dichlorides, $\mathrm{Ar}_{2} \mathrm{TeCl}_{2}(\mathrm{Ar}=p$-methoxyphenyl, $p$-hydroxyphenyl and 3-methyl-4-hydroxyphenyl), when treated with potassium salicylhydroxamate yield both 1:1 and 1:2 complexes of the type $\mathrm{Ar}_{2} \mathrm{TeCl}$.SHA and $\mathrm{Ar}_{2} \mathrm{Te}$.(SHA) $)_{2}$. These have been synthesized by the same procedure as for salicylhydroxamate of aryltellurium(IV) described above.

\section{Results and Discussion}

Tellurium tetrachloride when heated with anisole ${ }^{47-49}$, phenol $^{50}, o-\mathrm{cresol}^{51}(\mathrm{Ar}-\mathrm{H})$ appears to undergo Friedel-Crafts type condensation reaction whereby $\mathrm{TeCl}_{3}{ }^{+}$unit attacks a position para to the $-\mathrm{OH} /-\mathrm{OCH}_{3}$ groups in the aromatic rings, thus resulting in the formation of aryltellurium(IV) trichlorides and diaryltellurium(IV) dichlorides.

$$
\begin{gathered}
\mathrm{Ar}-\mathrm{H}+\mathrm{TeCl}_{4} \longrightarrow \mathrm{ArTeCl}_{3}+\mathrm{HCl} \\
2 \mathrm{Ar}-\mathrm{H}+\mathrm{TeCl}_{4} \longrightarrow \mathrm{Ar}_{2} \mathrm{TeCl}_{2}+2 \mathrm{HCl}
\end{gathered}
$$

Preparation of potassium salicylhydroxamate involves in two steps: first the preparation of ethyl ester of salicylic acid and then the potassium salt, which can be represented by following equations.

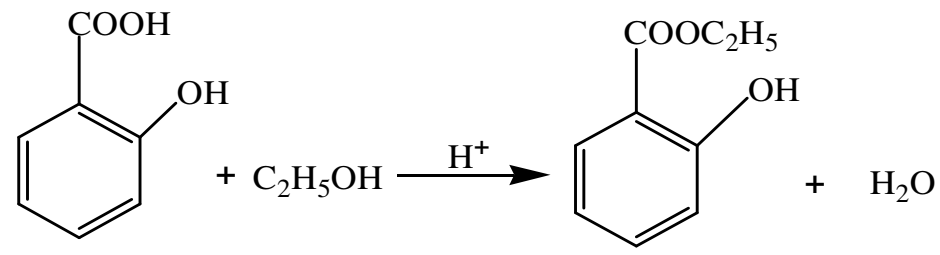




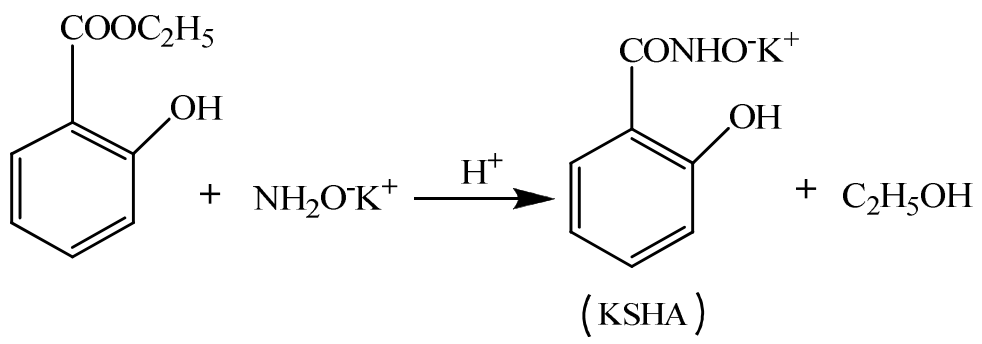

Potassium salicylhydroxamate (KSHA) reacts with aryltellurium(IV) trichlorides and diaryltellurium(IV) dichlorides in 1:1 and 2:1 molar ratios to yield the corresponding aryltellurium(IV) hydroxamate.

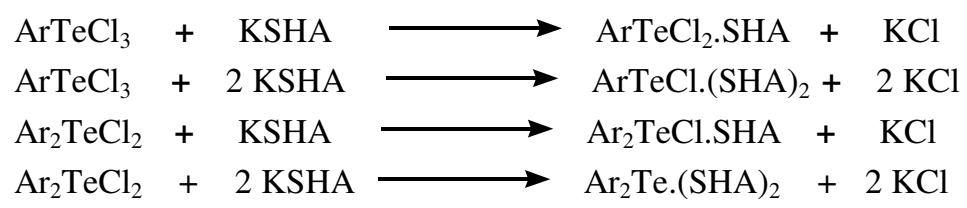

These new aryltellurium(IV) hydroxamates have been analysed for tellurium, chloride, carbon, hydrogen and nitrogen contents and the data along with their physical properties and yields are presented in Table 1.

\section{Conductance studies}

Molar conductance $\left(\Lambda_{\mathrm{M}}\right)$ data for aryltellurium(IV) hydroxamates in DMSO are compiled in Table 1 . The $\Lambda_{\mathrm{M}}$ values at $c a \cdot 10^{-3} \mathrm{M}$ predicts the weak to $1: 1$ electrolyte ${ }^{53,54}$ type behavior of these hydroxamates in DMSO, probably due to ionization into $\mathrm{ArTeCl}_{\mathrm{SHA}}{ }^{+}$ ArTe.(SHA $)_{2}{ }^{+} / \mathrm{Ar}_{2} \mathrm{Te} . \mathrm{SHA}^{+}$and $\mathrm{Cl}^{-}$in DMSO. The higher $\Lambda_{\mathrm{M}}$ values for $\mathrm{Ar}_{2} \mathrm{Te}$. $(\mathrm{SHA})_{2}$ may be due to steric factors and donor behavior of DMSO to result in probable dissociation into $\mathrm{Ar}_{2}$ Te.SHA.DMSO${ }^{+}$and $\mathrm{SHA}^{-}$ions.

\section{Infrared spectra}

The infrared spectra of the aryltellurium(IV) hydroxamates are quite complex and an attempt has therefore been made to identify the donor sites of hydroxamate ligand by comparing with those of parent aryltellurium(IV) chlorides and potassium hydroxamate, which illustrated clear differences.

The principal infrared absorption bands of KSHA are those due to $v_{(\mathrm{C}=\mathrm{O}),} v_{(\mathrm{C}-\mathrm{N})}, v_{(\mathrm{N}-\mathrm{O})}$ and $v_{(\mathrm{N}-\mathrm{H})}$ stretching vibrations of the hydroxamate group observed in the spectrum at $1606 \mathrm{~cm}^{-1}$, $1392 \mathrm{~cm}^{-1}, 999 \mathrm{~cm}^{-1}$ and $3287-3233 \mathrm{~cm}^{-1}$ respectively (Table 2).

The absorption band occurring at $1606 \mathrm{~cm}^{-1}$ in KSHA attributes to $v_{(\mathrm{C}=0)}$ mode, shifted to lower wave numbers and appeared at $1576-1588 \mathrm{~cm}^{-1}$ in aryltellurium(IV) hydroxamates. The absorption band due to $v_{(\mathrm{C}-\mathrm{N})}$ mode occurring at $1392 \mathrm{~cm}^{-1}$ in free KSHA has been found to shift towards higher region at $1453-1456 \mathrm{~cm}^{-1}$ in the complexes. The band at 3233-3288 $\mathrm{cm}^{-1}$ due to $v_{(\mathrm{N}-\mathrm{H})}$ mode in KSHA did not undergo any change, however could not be ascertained due to phenolic $\mathrm{OH}$ group in the aryltellurium moiety. This rules out the involvement of coordination through nitrogen atom. The sharp band occurring at $999 \mathrm{~cm}^{-1}$ in KSHA ascribed to $v_{(\mathrm{N}-\mathrm{O})}$ mode has been observed to move towards higher wave number and appeared at $1025-1032 \mathrm{~cm}^{-1}$ in aryltellurium(IV) salicylhydroxamates. 
Table 1. Analytical data, molar conductance and physical properties of aryltellurium(IV) salicylhydroxamates

\begin{tabular}{|c|c|c|c|c|c|c|c|c|c|c|}
\hline \multirow{2}{*}{ 总 } & \multirow{2}{*}{$\begin{array}{l}\text { Complex } \\
\text { (Ar) }\end{array}$} & \multirow{2}{*}{$\begin{array}{l}\text { Empirical Formula } \\
\text { (Formula Wt.) }\end{array}$} & \multirow{2}{*}{$\begin{array}{l}\text { Colour, } \\
\text { Yield, \% }\end{array}$} & \multirow{2}{*}{$\begin{array}{l}\text { M. Pt., } \\
{ }^{\circ} \mathrm{C} \\
\text { dec. }\end{array}$} & \multicolumn{5}{|c|}{ Analyses \% Found (Calculated) } & \multirow{2}{*}{$\begin{array}{c}\Lambda_{\mathrm{M}} \text { at } c a \cdot 10^{-3} \mathrm{M} \\
\mathrm{ohm}^{-1} \mathrm{~cm}^{2} \mathrm{~mol}^{-1} \\
\text { in DMSO }\end{array}$} \\
\hline & & & & & $\mathrm{C}$ & $\mathrm{H}$ & $\mathrm{N}$ & $\mathrm{Te}$ & $\mathrm{Cl}$ & \\
\hline I & $\begin{array}{c}\mathrm{ArTeCl}_{2} \cdot \mathrm{SHA} \\
(p-\text {-methoxyphenyl) }\end{array}$ & $\begin{array}{c}\mathrm{C}_{14} \mathrm{H}_{13} \mathrm{Cl}_{2} \mathrm{NO}_{4} \mathrm{Te} \\
(457.66)\end{array}$ & $\begin{array}{l}\text { Dull white } \\
\text { (92) }\end{array}$ & $75-78$ & $\begin{array}{c}36.44 \\
(36.71)\end{array}$ & $\begin{array}{c}2.72 \\
(2.84)\end{array}$ & $\begin{array}{c}2.86 \\
(3.06)\end{array}$ & $\begin{array}{l}28.70 \\
(27.87)\end{array}$ & $\begin{array}{l}15.25 \\
(15.51)\end{array}$ & 37.63 \\
\hline II & $\begin{array}{c}\text { ArTeCl.(SHA) } \\
(p \text {-methoxyphenyl) }\end{array}$ & $\begin{array}{c}\mathrm{C}_{21} \mathrm{H}_{19} \mathrm{ClN}_{2} \mathrm{O}_{7} \mathrm{Te} \\
(574.29)\end{array}$ & $\begin{array}{c}\text { Cream } \\
(90)\end{array}$ & $85-90$ & $\begin{array}{c}43.51 \\
(43.86)\end{array}$ & $\begin{array}{l}3.12 \\
(3.31)\end{array}$ & $\begin{array}{c}5.12 \\
(4.87)\end{array}$ & $\begin{array}{l}21.92 \\
(22.21)\end{array}$ & $\begin{array}{l}5.98 \\
(6.17)\end{array}$ & 42.31 \\
\hline III & $\begin{array}{c}\mathrm{ArTeCl}_{2} \cdot \mathrm{SHA} \\
\text { (p-hydroxyphenyl) }\end{array}$ & $\begin{array}{c}\mathrm{C}_{13} \mathrm{H}_{11} \mathrm{Cl}_{2} \mathrm{NO}_{4} \mathrm{Te} \\
(443.65)\end{array}$ & $\begin{array}{l}\text { Light grey } \\
\text { (94) }\end{array}$ & $74-76$ & $\begin{array}{c}34.72 \\
(35.14)\end{array}$ & $\begin{array}{c}2.79 \\
(2.48)\end{array}$ & $\begin{array}{c}3.35 \\
(3.16)\end{array}$ & $\begin{array}{c}28.52 \\
(28.76)\end{array}$ & $\begin{array}{c}15.74 \\
(16.00)\end{array}$ & 48.41 \\
\hline IV & $\begin{array}{c}\text { ArTeCl.(SHA) })_{2} \\
(p \text {-hydroxyphenyl) }\end{array}$ & $\begin{array}{c}\mathrm{C}_{20} \mathrm{H}_{17} \mathrm{ClN}_{2} \mathrm{O}_{7} \mathrm{Te} \\
(560.28)\end{array}$ & $\begin{array}{c}\text { Light } \\
\text { brown (95) }\end{array}$ & $78-80$ & $\begin{array}{c}42.52 \\
(42.82)\end{array}$ & $\begin{array}{c}3.24 \\
(3.03)\end{array}$ & $\begin{array}{c}4.98 \\
(5.00)\end{array}$ & $\begin{array}{c}22.43 \\
(22.77)\end{array}$ & $\begin{array}{c}6.40 \\
(6.33)\end{array}$ & 25.22 \\
\hline $\mathrm{V}$ & $\begin{array}{c}\mathrm{ArTeCl}_{2} \cdot \mathrm{SHA} \\
\text { (3-methyl-4- } \\
\text { hydroxyphenyl) }\end{array}$ & $\begin{array}{c}\mathrm{C}_{14} \mathrm{H}_{13} \mathrm{Cl}_{2} \mathrm{NO}_{4} \mathrm{Te} \\
(457.66)\end{array}$ & $\begin{array}{l}\text { Brown } \\
(85)\end{array}$ & $50-52$ & $\begin{array}{c}35.98 \\
(36.71)\end{array}$ & $\begin{array}{l}2.99 \\
(2.84)\end{array}$ & $\begin{array}{c}2.98 \\
(3.06)\end{array}$ & $\begin{array}{c}27.41 \\
(27.87)\end{array}$ & $\begin{array}{c}14.98 \\
(15.51)\end{array}$ & 48.71 \\
\hline VI & $\begin{array}{c}\text { ArTeCl.(SHA) } \\
\text { (3-methyl-4- } \\
\text { hydroxyphenyl) }\end{array}$ & $\begin{array}{c}\mathrm{C}_{21} \mathrm{H}_{19} \mathrm{ClN}_{2} \mathrm{O}_{7} \mathrm{Te} \\
(574.29)\end{array}$ & $\begin{array}{l}\text { Pale } \\
\text { yellow } \\
(82)\end{array}$ & $60-64$ & $\begin{array}{c}42.60 \\
(43.86)\end{array}$ & $\begin{array}{c}3.63 \\
(3.31)\end{array}$ & $\begin{array}{c}4.93 \\
(4.87)\end{array}$ & $\begin{array}{c}22.06 \\
(22.21)\end{array}$ & $\begin{array}{c}6.42 \\
(6.17)\end{array}$ & 39.15 \\
\hline VII & $\begin{array}{c}\mathrm{Ar}_{2} \mathrm{TeCl} \text {.SHA } \\
(p-\text {-methoxyphenyl) }\end{array}$ & $\begin{array}{c}\mathrm{C}_{21} \mathrm{H}_{20} \mathrm{ClNO}_{5} \mathrm{Te} \\
(529.29)\end{array}$ & $\begin{array}{c}\text { Cream } \\
(84)\end{array}$ & $\begin{array}{l}115- \\
120\end{array}$ & $\begin{array}{c}46.76 \\
(47.59)\end{array}$ & $\begin{array}{c}3.99 \\
(3.78)\end{array}$ & $\begin{array}{l}2.30 \\
(2.65)\end{array}$ & $\begin{array}{c}25.12 \\
(24.10)\end{array}$ & $\begin{array}{c}7.37 \\
(6.70)\end{array}$ & 25.83 \\
\hline VIII & $\begin{array}{c}\mathrm{Ar}_{2} \mathrm{Te} .(\mathrm{SHA})_{2} \\
(p \text {-methoxyphenyl })\end{array}$ & $\begin{array}{c}\mathrm{C}_{28} \mathrm{H}_{26} \mathrm{~N}_{2} \mathrm{O}_{8} \mathrm{Te} \\
(645.92)\end{array}$ & $\begin{array}{l}\text { Brown } \\
(87)\end{array}$ & $\begin{array}{l}110- \\
112\end{array}$ & $\begin{array}{c}52.87 \\
(52.00)\end{array}$ & $\begin{array}{c}4.43 \\
(4.02)\end{array}$ & $\begin{array}{c}4.17 \\
(4.33)\end{array}$ & $\begin{array}{c}19.07 \\
(19.75)\end{array}$ & - & 73.12 \\
\hline IX & $\begin{array}{c}\mathrm{Ar}_{2} \mathrm{TeCl} \text {.SHA } \\
(p \text {-hydroxyphenyl) }\end{array}$ & $\begin{array}{c}\mathrm{C}_{19} \mathrm{H}_{16} \mathrm{ClNO}_{5} \mathrm{Te} \\
(501.26)\end{array}$ & $\begin{array}{l}\text { Pink } \\
\text { (89) }\end{array}$ & $\begin{array}{l}100- \\
105\end{array}$ & $\begin{array}{c}44.71 \\
(45.47)\end{array}$ & $\begin{array}{c}3.46 \\
(3.19)\end{array}$ & $\begin{array}{c}3.12 \\
(2.79)\end{array}$ & $\begin{array}{c}26.39 \\
(25.45)\end{array}$ & $\begin{array}{c}7.23 \\
(7.08)\end{array}$ & 20.24 \\
\hline $\mathrm{X}$ & $\begin{array}{c}\mathrm{Ar}_{2} \mathrm{Te} .(\mathrm{SHA})_{2} \\
(p \text {-hydroxyphenyl) }\end{array}$ & $\begin{array}{c}\mathrm{C}_{26} \mathrm{H}_{22} \mathrm{~N}_{2} \mathrm{O}_{8} \mathrm{Te} \\
(617.89)\end{array}$ & $\begin{array}{l}\text { Orange } \\
(91)\end{array}$ & $86-88$ & $\begin{array}{c}49.99 \\
(50.48)\end{array}$ & $\begin{array}{c}3.98 \\
(3.56)\end{array}$ & $\begin{array}{c}4.67 \\
(4.53)\end{array}$ & $\begin{array}{l}21.01 \\
(20.65)\end{array}$ & - & 22.88 \\
\hline XI & $\begin{array}{c}\mathrm{Ar}_{2} \mathrm{TeCl} \text {.SHA } \\
\text { (3-methyl-4- } \\
\text { hydroxyphenyl) }\end{array}$ & $\begin{array}{c}\mathrm{C}_{21} \mathrm{H}_{20} \mathrm{ClNO}_{5} \mathrm{Te} \\
(529.29)\end{array}$ & $\begin{array}{l}\text { Red } \\
(78)\end{array}$ & $\begin{array}{l}120- \\
122\end{array}$ & $\begin{array}{c}47.65 \\
(47.59)\end{array}$ & $\begin{array}{c}3.60 \\
(3.78)\end{array}$ & $\begin{array}{c}2.12 \\
(2.65)\end{array}$ & $\begin{array}{c}25.22 \\
(24.10)\end{array}$ & $\begin{array}{c}6.36 \\
(6.70)\end{array}$ & 11.64 \\
\hline XII & $\begin{array}{c}\mathrm{Ar}_{2} \mathrm{Te} .(\mathrm{SHA})_{2} \\
\text { (3-methyl-4- } \\
\text { hydroxyphenyl) }\end{array}$ & $\begin{array}{c}\mathrm{C}_{28} \mathrm{H}_{26} \mathrm{~N}_{2} \mathrm{O}_{8} \mathrm{Te} \\
(645.92)\end{array}$ & $\begin{array}{l}\text { Grey } \\
(75)\end{array}$ & $\begin{array}{l}148- \\
150\end{array}$ & $\begin{array}{c}52.36 \\
(52.00)\end{array}$ & $\begin{array}{c}4.49 \\
(4.02)\end{array}$ & $\begin{array}{c}4.20 \\
(4.33)\end{array}$ & $\begin{array}{c}18.93 \\
(19.75)\end{array}$ & - & 71.19 \\
\hline
\end{tabular}


A shift in $v_{(\mathrm{C}=\mathrm{O})}$ mode to lower wave number and $v_{(\mathrm{N}-\mathrm{O})}$ mode to higher wave numbers are suggestive of bonding of salicylhydroxamate ion via oxygen atoms of carbonyl and hydroxylamine group ${ }^{19,23,55-58}$. The appearance of new weak bands around $283-297 \mathrm{~cm}^{-1}$ due to $v_{(\mathrm{Te}-\mathrm{O})}^{59-61}$ confirms the bonding of ligand to tellurium through the oxygen atoms. Also, independent assignment of $v_{\mathrm{O}-\mathrm{H}}$ (phenolic) could not be made due to presence of this group in hydroxyaryltellurium(IV) moiety i.e. ArTe or $\mathrm{Ar}_{2} \mathrm{Te}$.

\section{${ }^{1}$ H NMR spectra}

Proton magnetic resonance spectra of aryltellurium(IV) salicylhydroxamates are very complex and a lot of mixing of aryl proton singals of the salicylhydroxamate and aryltellurium(IV) moiety takes place, thus making the independent assignment almost impossible. The chemical shift data for the free salicylhydroxamic acid ${ }^{62}$ and aryltellurium(IV) salicylhydroxamates are complied in the Table 3.

Table 2. Important infrared absorption bands $\left(\mathrm{cm}^{-1}\right)$ of potassium salicylhydroxamate and aryltellurium(IV) hydroxamates

\begin{tabular}{ccccc}
\hline Compoud & $v_{(\mathrm{C}=\mathrm{O})}$ & $v_{(\mathrm{C}-\mathrm{N})}$ & $v_{(\mathrm{N}-\mathrm{O})}$ & $v_{(\mathrm{Te}-\mathrm{O})}$ \\
\hline KSHA & $1606 \mathrm{~s}$ & $1392 \mathrm{vs}$ & $999 \mathrm{~s}$ & - \\
I & $1580 \mathrm{~s}$ & $1454 \mathrm{~m}$ & $1029 \mathrm{~m}$ & $288 \mathrm{~W}$ \\
II & $1579 \mathrm{~s}$ & $1454 \mathrm{~m}$ & $1032 \mathrm{~m}$ & $297 \mathrm{~W}$ \\
III & $1577 \mathrm{~s}$ & $1454 \mathrm{~m}$ & $1032 \mathrm{~m}$ & $289 \mathrm{~W}$ \\
IV & $1577 \mathrm{~s}$ & $1454 \mathrm{~m}$ & $1032 \mathrm{~m}$ & $283 \mathrm{w}$ \\
V & $1576 \mathrm{~s}$ & $1453 \mathrm{~m}$ & $1031 \mathrm{~m}$ & $288 \mathrm{~W}$ \\
VI & $1577 \mathrm{~s}$ & $1454 \mathrm{~s}$ & $1032 \mathrm{~m}$ & $288 \mathrm{~W}$ \\
VII & $1583 \mathrm{~s}$ & $1454 \mathrm{~s}$ & $1028 \mathrm{~m}$ & $290 \mathrm{~W}$ \\
VIII & $1584 \mathrm{~s}$ & $1456 \mathrm{~m}$ & $1027 \mathrm{~m}$ & $290 \mathrm{~W}$ \\
IX & $1577 \mathrm{vs}$ & $1453 \mathrm{~s}$ & $1032 \mathrm{~m}$ & $288 \mathrm{~W}$ \\
X & $1577 \mathrm{vs}$ & $1453 \mathrm{~s}$ & $1032 \mathrm{~m}$ & $288 \mathrm{~W}$ \\
XI & $1588 \mathrm{vs}$ & $1454 \mathrm{~s}$ & $1025 \mathrm{~m}$ & $292 \mathrm{~W}$ \\
XII & $1588 \mathrm{vs}$ & $1454 \mathrm{~m}$ & $1026 \mathrm{~m}$ & $290 \mathrm{~W}$ \\
\hline
\end{tabular}

Table 3. ${ }^{1} \mathrm{H}$ NMR spectral data of aryltellurium(IV) salicylhydroxamates in DMSO- $\mathrm{d}_{6}$

\begin{tabular}{|c|c|}
\hline Compound & Chemical Shift, $\delta \mathrm{ppm}$ \\
\hline $\mathrm{C}_{6} \mathrm{H}_{4}(\mathrm{OH})$ & 6.82(q, 1H), 6.86(d, 1H), 7.35(q, 1H), 7.65(d, 1H), 9.32(s, 1H, -NOH), \\
\hline CONHOH & $11.43(\mathrm{~s}, 1 \mathrm{H},-\mathrm{NH}), 12.20(\mathrm{~s}, 1 \mathrm{H}$, phenolic $\mathrm{OH})$ \\
\hline I & $\begin{array}{l}3.87\left(\mathrm{~s}, 3 \mathrm{H},-\mathrm{OCH}_{3}\right), 6.81-8.38(\mathrm{~cm}, 8 \mathrm{H} \text {, aryl protons of ArTe and SHA }) \\
11.46(\mathrm{~s}, 1 \mathrm{H},-\mathrm{NH}), 12.40(\mathrm{bs}, 1 \mathrm{H}, \text { phenolic } \mathrm{OH} \text { of SHA })\end{array}$ \\
\hline II & $\begin{array}{l}3.83\left(\mathrm{~s}, 3 \mathrm{H},-\mathrm{OCH}_{3}\right), 6.81-8.39(\mathrm{~cm}, 12 \mathrm{H} \text {, aryl protons of ArTe and SHA }) \\
11.45(\mathrm{~s}, 2 \mathrm{H},-\mathrm{NH}), 12.30(\mathrm{bs}, 2 \mathrm{H}, \text { phenolic } \mathrm{OH} \text { of SHA })\end{array}$ \\
\hline III & $\begin{array}{l}\text { 6.83-7.81(cm, } 8 \mathrm{H} \text {, aryl protons of ArTe and SHA }), 8.24(\mathrm{~s}, 1 \mathrm{H}, \mathrm{OH} \text { of } \\
\text { ArTe }), 11.45(\mathrm{~s}, 1 \mathrm{H},-\mathrm{NH}), 10.09(\mathrm{bs}, 1 \mathrm{H}, \text { phenolic } \mathrm{OH} \text { of SHA })\end{array}$ \\
\hline IV & $\begin{array}{l}\text { 6.817-7.94(cm, } 12 \mathrm{H} \text {, aryl protons of ArTe and SHA }), 8.21(\mathrm{~s}, 1 \mathrm{H}, \mathrm{OH} \text { of } \\
\mathrm{ArTe}), 11.45(\mathrm{~s}, 2 \mathrm{H},-\mathrm{NH}), 12.264(\mathrm{bs}, 2 \mathrm{H} \text {, phenolic } \mathrm{OH} \text { of SHA })\end{array}$ \\
\hline V & $\begin{array}{l}2.16\left(\mathrm{~s}, 3 \mathrm{H},-\mathrm{CH}_{3}\right), 6.74-7.83(\mathrm{~cm}, 7 \mathrm{H}, \text { aryl protons of ArTe and SHA }), \\
8.11(\mathrm{~s}, 1 \mathrm{H}, \mathrm{OH} \text { of } \mathrm{ArTe}), 11.45(\mathrm{~s}, 1 \mathrm{H},-\mathrm{NH}), 9.97(\mathrm{bs}, 1 \mathrm{H}, \text { phenolic } \mathrm{OH} \text { of } \\
\text { SHA) }\end{array}$ \\
\hline
\end{tabular}




\begin{tabular}{|c|c|}
\hline VI & $\begin{array}{l}2.16\left(\mathrm{~s}, 3 \mathrm{H},-\mathrm{CH}_{3}\right), 6.82-7.81(\mathrm{~cm}, 11 \mathrm{H}, \text { aryl protons of } \mathrm{ArTe} \text { and } \mathrm{SHA}) \text {, } \\
8.11(\mathrm{~s}, 1 \mathrm{H}, \mathrm{OH} \text { of } \mathrm{ArTe}), 11.457(\mathrm{~s}, 2 \mathrm{H},-\mathrm{NH}), 10.10,12.31(\mathrm{bs}, 2 \mathrm{H} \text {, } \\
\text { phenolic } \mathrm{OH} \text { of SHA })\end{array}$ \\
\hline VII & $\begin{array}{l}3.83\left(\mathrm{~s}, 6 \mathrm{H},-\mathrm{OCH}_{3}\right), 6.815-8.008\left(\mathrm{~cm}, 12 \mathrm{H} \text {, aryl protons of } \mathrm{Ar}_{2} \mathrm{Te} \text { and }\right. \\
\text { SHA }), 11.47(\mathrm{~s}, 1 \mathrm{H},-\mathrm{NH}), 12.296(\mathrm{~s}, 1 \mathrm{H}, \text { phenolic } \mathrm{OH} \text { of SHA })\end{array}$ \\
\hline VIII & $\begin{array}{l}3.82\left(\mathrm{~s}, 6 \mathrm{H},-\mathrm{OCH}_{3}\right), 6.816-7.977\left(\mathrm{~cm}, 16 \mathrm{H} \text {, aryl protons of } \mathrm{Ar}_{2} \mathrm{Te} \text { and }\right. \\
\text { SHA }), 11.48(\mathrm{~s}, 2 \mathrm{H},-\mathrm{NH}), 12.29(\mathrm{~s}, 2 \mathrm{H}, \text { phenolic } \mathrm{OH} \text { of SHA })\end{array}$ \\
\hline IX & $\begin{array}{l}\left.\text { 6.767-7.879(cm, } 12 \mathrm{H} \text {, aryl protons of } \mathrm{Ar}_{2} \mathrm{Te} \text { and } \mathrm{SHA}\right), 9.33(\mathrm{~s}, 2 \mathrm{H}, \mathrm{OH} \text { of } \\
\left.\mathrm{Ar}_{2} \mathrm{Te}\right), 11.46(\mathrm{~s}, 1 \mathrm{H},-\mathrm{NH}), 12.286(\mathrm{~s}, 1 \mathrm{H} \text {, phenolic } \mathrm{OH} \text { of } \mathrm{SHA})\end{array}$ \\
\hline$X$ & $\begin{array}{l}\left.\text { 6.774-7.828(cm, } 16 \mathrm{H} \text {, aryl protons of } \mathrm{Ar}_{2} \mathrm{Te} \text { and } \mathrm{SHA}\right), 9.37(\mathrm{~s}, 2 \mathrm{H}, \mathrm{OH} \text { of } \\
\left.\mathrm{Ar}_{2} \mathrm{Te}\right), 11.467(\mathrm{~s}, 2 \mathrm{H},-\mathrm{NH}), 12.257(\mathrm{~s}, 2 \mathrm{H} \text {, phenolic } \mathrm{OH} \text { of } \mathrm{SHA})\end{array}$ \\
\hline XI & $\begin{array}{l}2.53\left(\mathrm{~s},-\mathrm{CH}_{3}\right), 6.218-7.757\left(\mathrm{~cm} \text {, aryl protons of } \mathrm{Ar}_{2} \mathrm{Te} \text { and } \mathrm{SHA}\right), 8.20(\mathrm{~s} \text {, } \\
\left.\mathrm{OH} \text { of } \mathrm{Ar}_{2} \mathrm{Te}\right) \text {, poor solubility, spectra not well resolved }\end{array}$ \\
\hline XII & $\begin{array}{l}2.51\left(\mathrm{~s},-\mathrm{CH}_{3}\right), 6.628-7.749\left(\mathrm{~cm} \text {, aryl protons of } \mathrm{Ar}_{2} \mathrm{Te} \text { and } \mathrm{SHA}\right), 8.26(\mathrm{~s} \text {, } \\
\left.\mathrm{OH} \text { of } \mathrm{Ar}_{2} \mathrm{Te}\right), \text { spectra not well resolved due to very poor solubility }\end{array}$ \\
\hline
\end{tabular}

$s=$ singlet,$d=$ doublet,$q=$ quartet, $\mathrm{cm}=$ complex multiplet, $\mathrm{bs}=$ broad singlet

Free salicylhydroxamic acid shows four separate singals due to aryl proton at $6.82,6.86$, 7.35 and $7.65 \delta \mathrm{ppm}$. The hydroxamic $\mathrm{N}-\mathrm{H}$ and $\mathrm{O}-\mathrm{H}$ protons resonate at 11.43 and $9.32 \delta \mathrm{ppm}$ respectively, and phenolic $\mathrm{OH}$ at $12.20 \delta \mathrm{ppm}$ as singlets. The hydroxamic $\mathrm{OH}$ singlet at $9.32 \delta \mathrm{ppm}$ disappears in all the complexes indicating thereby the deprotonation of $-\mathrm{NOH}$ proton and subsequently linkage of tellurium with this oxygen atom. The N-H proton signal at $\delta 11.43$ in free salicylhydroxamic acid, also is observed in all the salicylhydroxamate complexes, confirming its non participation in the bonding with tellurium atom. The broad singlet around $12 \mathrm{ppm}$ indicates that phenolic $\mathrm{OH}$ of salicylhydroxamate is not involved in bonding with the tellurium. The spectra of complexes of bis(3-methyl-4-hydroxyphenyl)tellurium(IV) are not well resolved due to very poor solubility in $\mathrm{DMSO}_{-} \mathrm{d}_{6}$.

Further, the aryl protons of aryltellurium(IV), diaryltellurium(IV) and salicylhydroxamate groups exhibit a lot of overlapping of signals and are observed as complex multiplet in the region 6.218-8.39 $\delta \mathrm{ppm}$, as observed in ${ }^{1} \mathrm{H}$ NMR Spectra of organotin(IV) complexes of hydroxamic acids ${ }^{19}$. Also, a careful examination of ${ }^{1} \mathrm{H}$ NMR Spectra of hydroxamate complexes reveal the shielding of aryl protons of $\mathrm{ArTe}_{\mathrm{An}} \mathrm{Te}$ compared ${ }^{50,51,63,64}$ to $\mathrm{ArTeCl}_{3} / \mathrm{Ar}_{2} \mathrm{TeCl}_{2}$ and deshielding of protons of SHA due to flow of electron density from the ligand to the aryltellurium moiety as a result of complexation.

Thus, on the basis of infrared and proton magnetic resonance spectral studies it may be concluded that $\mathrm{SHA}^{-}$acts as a bidentate $(O, O)$ ligand involving the hydroxamate (-NHO) and carbonyl oxygens, giving rise to penta coordinated tellurium complexes in $\mathrm{ArTeCl}_{2} . \mathrm{SHA}$ and $\mathrm{Ar}_{2} \mathrm{TeCl}$ SHA and hexa coordinated in $\mathrm{ArTeCl}$.(SHA $)_{2}$ and $\mathrm{Ar}_{2} \mathrm{Te}$.(SHA $)_{2}$. The proposed structures (Figure 1) are as below:<smiles>Oc1ccccc1C1=NO[Te](Cl)(Cl)O1</smiles>

$\mathrm{ArTeCl}_{2}$.SHA<smiles></smiles>

ArTeCl.(SHA) 
<smiles>Oc1ccccc1C1NO[Ge]([Al])(Cl)O1</smiles>

$\mathrm{Ar}_{2} \mathrm{TeCl}$.SHA

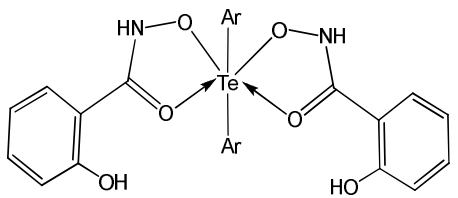

$\mathrm{Ar}_{2}$ Te.(SHA)

Figure 1. Proposed structure of complexes

\section{Antimicrobial activity}

All the newly synthesized complexes were investigated for their in vitro antimicrobial potential against gram positive bacteria ( $S$. aureus and B. subtilis), gram negative bacterium (E. coli) and fungal strains (C. albicans and A. niger) by tube dilution method. The minimum inhibitory concentration (MIC) values are presented in the Table 4.

Table 4. Minimum inhibitory concentration of potassium salicylhydroxamate and aryltellurium(IV) hydroxamates.

\begin{tabular}{cccccc}
\hline \multirow{2}{*}{ Compound } & \multicolumn{5}{c}{ MIC, $\mu \mathrm{g} / \mathrm{mL}$} \\
\cline { 2 - 6 } & S. aureus. & B. subtilis. & E. coli & A. niger & C. albicans \\
\hline KSHA & 12.5 & 12.5 & 12.5 & 12.5 & 6.25 \\
I & 12.5 & 12.5 & 6.25 & 6.25 & 6.25 \\
II & 25 & 12.5 & 12.5 & 6.25 & 6.25 \\
III & 12.5 & 12.5 & 6.25 & 12.5 & 12.5 \\
IV & 12.5 & 25 & 6.25 & 12.5 & 12.5 \\
V & 12.5 & 12.5 & 6.25 & 12.5 & 6.25 \\
VI & 12.5 & 12.5 & 6.25 & 6.25 & 6.25 \\
VII & 12.5 & 25 & 6.25 & 6.25 & 6.25 \\
VIII & 12.5 & 12.5 & 12.5 & 6.25 & 6.25 \\
IX & 12.5 & 25 & 12.5 & 6.25 & 6.25 \\
X & 12.5 & 25 & 12.5 & 6.25 & 6.25 \\
XI & 12.5 & 12.5 & 12.5 & 6.25 & 6.25 \\
XII & 12.5 & 12.5 & 12.5 & 6.25 & 6.25 \\
\hline
\end{tabular}

The MIC data reveal that the aryltellurium(IV) hydroxamate complexes and potassium hydroxamate possess more biocidal activity than the precursor salicylhydroxamaic $\mathrm{acid}^{22}$, which shows MIC in the range 66-88 $\mu \mathrm{g} / \mathrm{mL}$.

\section{Conclusion}

Aryltellurium(IV) trichlorides and diaryltellurium(IV) dichlorides upon reaction with potassium salicylhydroxamate in different molar ratios yield the 1:1 and 1:2 type complexes, whereby chloride is exchanged with salicylhydroxamate group. The hydroxamate complexes have been characterized by elemental analyses, conductance measurement, infrared and proton magnetic resonance spectral studies. The salicylhydroxamate in these complexes functions as a bidentate ligand through carbonyl and hydroxamate oxygen atoms. The complexes have been observed to possess appreciable antimicrobial activity against bacteria and fungi.

\section{Acknowledgement}

The authors are grateful to M. D. University, Rohtak for providing the necessary facilities. One of the authors (SC) is also thankful to UGC New Delhi for providing a fellowship. We also thank SAIF, Panjab University Chandigarh for providing the CHN analyses and spectral data. 


\section{References}

1. Raymod K N, Coord Chem Rev., 1990, 105, 135-153; DOI:10.1016/00108545(90)80020-T

2. Stemmler A J, Kampf J W, Kirk M L and Pecoraro V L, J Am Chem Soc., 1995, 117(23), 6368-6369; DOI:10.1021/ja00128a031

3. Pearson M A, Michel L O, Hausinger R P and Karplus P A, Biochemistry, 1997, 36(26), 8164-8172; DOI:10.1021/bi970514j

4. Parvathy S, Hussain I, Karran E H, Turner A J and Hooper N M, Biochemistry, 1998, 37(3), 1680-1685; DOI:10.1021/bi972034y

5. Pikul S, McDow Dunham K L, Almstead N G, De B, Natchus M G, Anastasio M U, McPhail S J, Snider C E, Taivo Yo, Chen L, Dunaway C M, Gu F and Mieling G E, $J$ Med Chem., 1999, 42(1), 87-94; DOI:10.1021/jm980142s

6. Muri E M, Nieto M J, Sindelar R D and Williamson J S, Curr Med Chem., 2002, 9(17), 1631-1653; DOI:10.2174/0929867023369402

7. Reddy A S, Kumar M S and Reddy G R, Tetrahedron Lett., 2000, 41(33), 6285-6288; DOI:10.1016/S0040-4039(00)01058-3

8. Steward W P and Thomas A L, Expert Opin Invest Drugs, 2000, 9(12), 2913-2922; DOI:10.1517/13543784.9.12.2913

9. Steward W P, Cancer Chemother Pharmacol., 1999, 43(Suppl 1), S56-S60; DOI: $10.1007 / \mathrm{s} 002800051099$

10. Domingo J L, Reprod Toxicol., 1998, 12(5), 499-510; DOI:10.1016/S08906238(98)00036-7

11. Turcot I, Stintzi A, Xu J and Raymond K N, J Biol Inorg Chem., 2000, 5(5), 634-641; DOI: $10.1007 / \mathrm{s} 007750000149$

12. Miller M J, Chem Rev., 1989, 89(7), 1563-1579; DOI:10.1021/cr00097a011

13. Cabantchik Z I, Parasitol Today, 1995, 11(2), 74-78; DOI:10.1016/01694758(95)80123-5

14. Mizukami S and Nagata K, Coord Chem Rev., 1968, 3(2), 267-278; DOI:10.1016/S0010-8545(00)80117-4

15. Chatterjee B, Coord Chem Rev., 1978, 26(3), 281-303; DOI:10.1016/S00108545(00)80350-1

16. Ugwu D I, Ezema B E, Eze F U, Ayogu J I, Ezema C G and Ugwaja D I, Am J Org Chem., 2014, 4, 26-51.

17. Codd R, Coord Chem Rev., 2008, 252(12-14), 1387-1408; DOI:10.1016/j.ccr.2007.08.001

18. Shang X, Cui J, Wu J, Pombeiro A J L and Li Q, J Inorg Bio Chem., 2008, 102(4), 901-909; DOI:10.1016/j.jinorgbio.2007.12.010

19. Naqeebullah, Farina Y, Chan K M, Mun L K, Rajab N F and Ooi T C, Molecules, 2013, 18(7), 8696-8711; DOI:10.3390/molecules18078696

20. Hall M D, Failes T W, Hibbs D E and Hambley T W, Inorg Chem., 2002, 41, 1223

21. Failes T W and Hambley T W, Aust J Chem., 2003, 56, 45.

22. Fazary A E, Bull Chem Soc Ethiop., 2014, 28(3), 393-402; DOI:10.4314/bcse.v28i3.8

23. Sharma S and Sharma N, Der Chemica Sinica, 2013, 4(3), 108-119.

24. Khairy E M, Shoukry M M, Khalil M M and Mohamed M M A, Trans Met Chem., 1996, 21(2), 176-180; DOI:10.1007/BF00136551

25. Sharma R and Sharma N, J Therm Anal Calorim., 2012, 110(2), 539-543.

26. Henderson W, Evans C, Nicholson B K and Fawcett, Dalton Trans., 2003, 26912697; DOI:10.1039/B303367H 
27. Hall M D, Failes T W, Hibbs D E and Hambley T W, Inorg Chem., 2002, 41(5), 1223-1228; DOI:10.1021/ic010760q

28. Wynne K J and Pearson P S, Inorg Chem., 1971, 10(12), 2735-2739; DOI:10.1021/ic50106a022

29. Wynne K J and Pearson P S, J Chem Soc Commun., 1970, 556.

30. Wynne K J, Clark A J and Berg M, J Chem Soc Dalton Trans., 1972, 2370-2374; DOI:10.1039/DT9720002370

31. Clark E R, Collet A J and Naik D G, J Chem Soc Dalton Trans., 1973, 1961-1962; DOI:10.1039/DT9730001961

32. Berg M C, Diss Abstr Int., 1972, 33, 2982.

33. Srivastava T N, Singh M and Singh H B, Indian J Chem., 1982, 21A, 307-309.

34. Srivastava T N, Srivastava R C and Srivastava M, Indian J Chem., 1982, 21A, 539.

35. Srivastava T N, Srivastava R C and Srivastava V K, J Indian Chem Soc., 1983, 60(9), 891-892.

36. Garad M V, Polyhedron, 1985, 4(8), 1353-1355; DOI:10.1016/S02775387(00)86963-6

37. Verma K K and Reena K K, Synth React Inorg Met Org Chem., 1999, 29(3), 499512; DOI:10.1080/00945719909349465

38. Verma K K, Dahiya R and Soni D, Synth React Inorg Met Org Chem., 1999, 29(6), 1033-1052; DOI:10.1080/00945719909349509

39. Verma K K and Dahiya R, Synth React Inorg Met Org Chem., 1999, 29(7), 12991314; DOI:10.1080/00945719909349529

40. Verma K K and Reena, Phosphorus, Sulfur Silicon Related Elements, 1999, 148(1), 227-2134; DOI:10.1080/10426509908037013

41. Verma K K and Seema, Int J Chem Sci., 2008, 6(1), 371-380.

42. Srivastava S, Soni D K and Gupta H S, J Indian Chem Soc., 1996, 73, 255.

43. Narwal J K, Chhabra S, Malik R K, Garg S and Verma K K, Oriental J Chem., 2013, 29(4), 1339-1349.

44. Chhabra S and Verma K K, J Chem Pharm Res., 2010, 2(4), 569-575.

45. Vogel A I, A Test Book of Organic Chemistry, $3^{\text {rd }}$ Edn., Longman, London, 1975.

46. Weissberger A, Ed., Technique of Organic Chemistry, Vol. 7, $2^{\text {nd }}$ Edn., Interscience Publishers, Inc. N. Y., 1967.

47. Morgan G T and Kellet R E, J Chem Soc., 1926, 129, 1080-1088; DOI:10.1039/JR9262901080

48. Petragnani $\mathrm{N}$ and Stefani $\mathrm{H}$ A, Tellurium in Organic Chemistry, $2^{\text {nd }}$ Edn., Academic Press, London, 2007, 67, 76.

49. Bergman J, Tetrahedron, 1972, 28(12), 3323-3331; DOI:10.1016/S00404020(01)93674-9

50. Khandelwal B L, Kumar K and Berry F J, Inorg Chim Acta, 1981, 47, 135-137; DOI:10.1016/S0020-1693(00)89319-6

51. Khandelwal B L, Kumar K and Raina K, Synth React Inorg Met Org Chem., 1981, 11(1), 65-78; DOI:10.1080/00945718108059276

52. Hauser C R and Renfrow W B, Org Synth Coll., 1943, 2, 67; DOI: 10.15227/orgsyn.019.0015

53. Geary W J, Coord Chem Rev., 1971, 7(1), 81-122; DOI:10.1016/S00108545(00)80009-0

54. Greenwood N N, Straughan B P and Wilson A E, J Chem Soc A: 1968, 2209-2212; DOI:10.1039/J19680002209 
55. Shahid S, Ali S, Hussain M, Mazhar M, Mahmood S and Rehman S, Turk J Chem., 2002, 26(4), 589-598.

56. Selbin J, Coord Chem Rev., 1966, 1(3), 293-314; DOI:10.1016/S00108545(00)80142-3

57. Sharma N, Kanwar S S, Gupta R, Kumari L and Sharma R, Bull Chem Soc Jpn., 2012, 85(12), 1310-1317; DOI:10.1246/bcsj.20120066

58. Banerjee T K, Brahma S K and Bag S P, Ind J Chem., 1992, 31A, 202.

59. Verma K K, Soni D and Verma S, Phosphorus Sulfur Silicon, 2000, 166(1), 231-241; DOI:10.1080/10426500008076544

60. Pant B C, McWhinnie W R and Dance N S, J Organmetal Chem., 1973, 63, 305-310; DOI:10.1016/S0022-328X(73)80043-9

61. Srivastava T N and Singh J D, Indian J Chem., 1987, 26A, 260.

62. Kaczor A and Proniewicz L M, J Mol Stru (Theochem), 2003, 640(1-3), 133-141; DOI:10.1016/j.theochem.2003.08.119

63. Raina K and Khandelwal B L, Indian J Chem., 1976, 14A, 63.

64. Berry F J, Kustan E H, Roshani M and Smith B C, J Organometal Chem., 1975, 99, 115-117; DOI:10.1016/S0022-328X(00)86367-6 